\title{
Private Payer and Medicare Coverage for Circulating Tumor DNA Testing: A Historical Analysis of Coverage Policies From 2015 to 2019
}

\author{
Michael P. Douglas, MS1; Stacy W. Gray, MD², and Kathryn A. Phillips, PhD ${ }^{1,4,5}$
}

\section{ABSTRACT}

Background: Clinical adoption of the sequencing of circulating tumor DNA (ctDNA) for cancer has rapidly increased in recent years. This sequencing is used to select targeted therapy and monitor nonresponding or progressive tumors to identify mechanisms of therapeutic resistance. Our study objective was to review available coverage policies for cancer ctDNA-based testing panels to examine trends from 2015 to 2019. Methods: We analyzed publicly available private payer policies and Medicare national coverage determinations and local coverage determinations (LCDs) for ctDNA-based panel tests for cancer. We coded variables for each year representing policy existence, covered clinical scenario, and specific ctDNA test covered. Descriptive analyses were performed. Results: We found that $38 \%$ of private payer coverage policies provided coverage of ctDNA-based panel testing as of July 2019. Most private payer policy coverage was highly specific: $87 \%$ for non-small cell lung cancer, $47 \%$ for EGFR gene testing, and $79 \%$ for specific brand-name tests. There were 8 final, 2 draft, and 2 future effective final LCDs (February 3 and March 15, 2020) that covered non-FDA-approved ctDNA-based tests. The draft and future effective LCDs were the first policies to cover pan-cancer use. Conclusions: Coverage of ctDNA-based panel testing for cancer indications increased from 2015 to 2019. The trend in private payer and Medicare coverage is an increasing number of coverage policies, number of positive policies, and scope of coverage. We found that Medicare coverage policies are evolving to pancancer uses, signifying a significant shift in coverage frameworks. Given that genomic medicine is rapidly changing, payers and policymakers (eg, guideline developers) will need to continue to evolve policies to keep pace with emerging science and standards in clinical care.

J Natl Compr Canc Netw 2020;18(7):866-872 doi: $10.6004 /$ jnccn.2020.7542

\footnotetext{
${ }^{1}$ Department of Clinical Pharmacy, UCSF Center for Translational and Policy Research on Personalized Medicine (TRANSPERS), San Francisco; ${ }^{2}$ Department of Population Science, and ${ }^{3}$ Department of Medical Oncology and Therapeutics Research, City of Hope, Duarte; and ${ }^{4}$ UCSF Philip R. Lee Institute for Health Policy, and ${ }^{5}$ UCSF Helen Diller Family Comprehensive Cancer Center, San Francisco, California.
}

\section{Background}

In recent years, there has been a rapid increase in the use of blood-based circulating tumor DNA (ctDNA) sequencing tests for cancer, with the first commercially available test offered by Guardant Health in 2014. Socalled liquid biopsy tests are used to select targeted therapy and monitor nonresponding or progressive tumors. Although most somatic (tumor) testing is performed on resected biopsy specimens, ctDNA tests can be used at the time of a cancer diagnosis to identify genomic alterations that may be effectively treated using targeted therapies. Furthermore, they are frequently used after a patient experiences disease progression on targeted therapy to determine the mechanism of therapeutic resistance, and after definitive therapy to detect recurrence. ${ }^{1-9}$ Blood-based assays are particularly valuable when testing needs to be performed rapidly and when tissue-based samples are unavailable.

The objective of this study was to review coverage policies for ctDNA-based testing panels in cancer to examine trends from 2015 to 2019, with 2015 being the first instance of an available payer coverage policy. Payer coverage for genomic medicine continues to be an important determinant of access to testing, including whether patients are tested and how they are tested. These types of coverage decisions are a key element in care, because testing and the subsequent use of targeted therapy ultimately influence patients' clinical outcomes. ${ }^{10-13}$ This study is the first to analyze current coverage of private and Medicare policies for ctDNA-based panel tests and to provide historical analysis.

The growth and adoption of ctDNA-based testing for cancer is expected to increase, as is the number of genes included in the test (eg, the Guardant360 test, which included 54 genes in 2014, now includes 73 genes in 2020). ${ }^{14}$ In addition, similar liquid biopsy techniques are

See JNCCN.org for supplemental online content. 
being applied beyond targeting cancer treatments to the early detection of cancer, which will further increase the scope of these tests as their use expands to healthy populations. In addition to providing tumor information, many of these assays may identify nontumor germline cell-free DNA (cfDNA) (eg, BRCA1/2 mutations), but reporting of such findings is inconsistent across laboratories. ${ }^{15}$ Thus, our study addresses broader important clinical and policy issues that are emerging. Our systematic assessment of coverage policies provides greater insight into the development of coverage policies and underscores how payers make coverage decisions over time.

\section{Methods}

We analyzed private commercially available payer coverage policies, Medicare (national coverage determinations [NCDs]), and Medicare Administrative Contractor (MAC) coverage policies (local coverage determinations [LCDs]) for ctDNA-based panel tests for cancer from January 1, 2015 through July 1, 2019. Definitions of terms are provided in supplemental eAppendix 1, available with this article at JNCCN.org.

\section{Data Sources and Payer Cohorts}

We used the Canary Insights Database (supplemental eAppendix 2) as the source of private policies and the Centers for Medicare \& Medicaid Services (CMS) coverage database for Medicare NCDs and LCDs (https:// www.cms.gov/medicare-coverage-database/search/ advanced-search.aspx). The Canary Insights Database (http://canaryinsights.com/) is a medical policy library containing $>40,000$ medical policies from $>200$ commercial payers and links to public payer policies with $>100,000$ members.

The private payer cohort was determined by including all payers that had a positive or negative coverage policy for ctDNA in the Canary Insights Database. The Medicare payer cohort was determined by reviewing all local and national Medicare policies on the CMS website with a positive coverage policy for ctDNA. ${ }^{16}$ Medicare has not issued negative coverage policies for these types of tests.

\section{Types of Coverage Policies}

\section{Private Payer}

Private policies are coverage policies issued by private companies for commercially available plans. They do not cover Medicare Advantage or payers that do not make their policies publicly available (eg, Kaiser Permanente).

\section{Medicare}

Medicare policies are coverage policies issued directly from CMS in the form of NCDs and by MACs in the form of LCDs. Before they are finalized, NCDs and LCDs are first issued as drafts. Draft policies are available for a public comment period and revision before the issuance of a Final NCD or LCD. There are 7 MACs (in 12 jurisdictions) in the United States that issue relevant coverage policies.

\section{Search Strategy and Policy Selection}

Policy titles and text were individually screened to determine whether they met inclusion criteria. We included policies that specifically addressed ctDNA-based panels as a clinical diagnostic test and excluded policies that addressed single-gene ctDNA testing (eg, EGFR, $A L K$, $B R A F$ ) that did not include a provision for ctDNA-based panel testing (supplemental eAppendix 3).

\section{Private Payers}

We searched the Canary Insights Database with the terms "liquid biopsy" and "circulating tumor DNA." We confirmed that these terms captured all relevant policies from January 1, 2015 through July 1, 2019 (supplemental eAppendix 3).

\section{Medicare}

We searched the CMS database for NCDs and LCDs with the terms "liquid biopsy" and "circulating tumor DNA" using the "all states" and "entire document" functions. We confirmed that these terms captured all relevant policies from January 1, 2018 (the date of the first positive policy) through July 1, 2019.

\section{Variables and Data Abstraction Policies}

We chose variables that described the structure of the policy (eg, how the policy was written, whether the policy was developed by a third party). For positive coverage policies, we chose variables that described how the coverage was worded, which cancers were included, if and how monitoring was included, and which specific ctDNA tests were covered. Specific variables that were coded for private and Medicare policies are listed in supplemental eTable 1.

We coded variables for private and Medicare policies for 2017 through 2019. We also coded whether a policy existed for 2015 to 2016 and if it provided coverage (as none provided coverage, no additional variables were coded). Data were independently coded by 2 individuals, and discrepancies were resolved by discussion. We described trends but did not statistically assess differences. Trends examined included the changes to policies (eg, noncoverage to coverage) and detailed coverage conditions (eg, test, genes, types of cancer).

\section{Results}

As of mid-2019, a total of 65 private payers and 4 MACs had published policies about ctDNA. We found a shift in private payers from no coverage in 2016 to $38 \%$ coverage 
in 2019 and a shift in Medicare policies from no LCDs for the use of ctDNA-based panel tests in cancer indications in 2017 to 8 final LCDs, 2 draft LCDs, and 2 future effective final LCD (February 3 and March 15, 2020) in 2019. From 2017 to 2019, policies increased in scope regarding the number of cancers included (from nonsmall cell lung cancer [NSCLC] to 12 solid/hematologic cancers) and from a single gene (EGFR) to 73 genes (eg, the Guardant360 test).

Specifically, we found that $38 \%(28 / 73)$ of private payer coverage policies provided coverage of ctDNAbased panel testing for some clinical indications as of July 1, 2019 (Table 1). Positive coverage policies were from 28 payers, of which 22 were Blue Cross Blue Shield Association (BCBSA) plans, and negative coverage policies were from 45 payers, of which 25 were BCBSA plans. Of the 200 payers in the Canary Insights Database, 33\% of private payers had explicitly positive or negative coverage policies.

Of the positive policies, $87 \%(24 / 28)$ were for NSCLC only (Table 2$)$ and nearly half $(47 \% ; 13 / 28)$ were written for testing EGFR gene analysis only (Table 3); 79\% (22/28) named coverage only for specific tests (cobas EGFR Mutation Test v2, Guardant360, or OncoBEAM) (Tables 2 and 3). Furthermore, of all positive policies, 11\% (3/28) specifically stated coverage for monitoring (eg, no prior testing and progression, or progression on tyrosine kinase inhibitors) (Table 2). Descriptions of all brand-named tests are provided in supplemental eAppendix 4 .

We found wide variation in how payers structured their policies-that is, whether the policy focused on the technology generally or on a specific cancer or test (Table 4). Private payers used 9 different approaches; the most common structure was to organize their policy for liquid biopsy tests, including both ctDNA and circulating tumor cell tests (36\%). Conversely, Medicare used 2 different approaches. The most common structure used by Medicare payers was to organize their policy based first on a specific named test (100\%) (eg, Guardant360 or InVisionFirst) and then narrowly on ctDNA NSCLC tests (67\%).

There were no NCD policies explicitly providing coverage for ctDNA-based panel tests. Although the NCD on sequencing for advanced cancer ${ }^{1}$ included ctDNA-based panel test coverage if the test was FDA-approved, there are currently no FDA-approved ctDNA-based panel tests.

Although there is no national coverage policy, MACs can provide coverage for ctDNA-based tests with the issuance of an LCD. We found $12 \mathrm{draft}$ or final LCDs (67\% final and $33 \%$ draft) covering ctDNA-based panel tests (Table 1) from 4 MACs (out of 7). Four MACs issued final LCD policies in 2018 for the Guardant360 ctDNA-based panel test for NSCLC. The same 4 MACs issued final LCD policies in 2019 for the InVisionFirst ctDNA-based panel test for NSCLC (Table 2). Between March and May 2019, the same 4 MACs issued draft LCD policies (Table 1) that will cover use of Guardant360 in ctDNA-based panel testing in 12 solid tumors, in addition to the already approved coverage policy for NSCLC (Table 2), when finalized. On December 19, 2019, a final LCD was issued by Palmetto GBA that finalized the LCD effective February 3, 2020, with a key addition that "other liquid biopsies will be covered for the same indications if they display similar performance in their intended used applications to Guardant360." 17

The first instance of a positive coverage policy was by Blue Cross Blue Shield Massachusetts in September 2017 for the Guardant360 ctDNA-based panel test for NSCLC, including the use of monitoring (data not shown). In 2018, there was a sharp increase in coverage for both private payers and Medicare, especially after the NCD on sequencing for advanced cancer ${ }^{1}$ was issued in March 2018.

Table 1. Coverage Policies for ctDNA

\begin{tabular}{|c|c|c|c|c|c|c|c|c|}
\hline Payer & 2015 & 2016 & 2017 & $\begin{array}{l}\text { Type of Cancers } \\
\text { Covered } \\
2017(n)\end{array}$ & 2018 & $\begin{array}{l}\text { Type of Cancers } \\
\text { Covered } 2018(n)^{b}\end{array}$ & 2019 & $\begin{array}{l}\text { Type of Cancers } \\
\text { Covered } 2019(n)^{b}\end{array}$ \\
\hline Private & $\begin{array}{l}0 / 6 \text { positive } \\
(0 \%)\end{array}$ & $\begin{array}{l}0 / 28 \text { positive } \\
(0 \%)\end{array}$ & $\begin{array}{c}\text { 1/42 positive } \\
\text { (3\%) }\end{array}$ & $\begin{array}{l}1 \text { lung cancer } \\
\text { only }\end{array}$ & $\begin{array}{l}13 / 66 \\
\text { positive } \\
(20 \%)\end{array}$ & $\begin{array}{c}11 \text { lung cancer only; } \\
2 \text { solid and } \\
\text { hematologic } \\
\text { malignancies }\end{array}$ & $\begin{array}{c}\text { 28/73 positive } \\
\text { (38\%) }\end{array}$ & $\begin{array}{c}24 \text { lung cancer only; } \\
4 \text { solid and hematologic } \\
\text { malignancies }\end{array}$ \\
\hline Medicare $^{a}$ & 0 positive & 0 positive & 0 positive & N/A & 4 positive & $\begin{array}{c}4 \text { lung cancer } \\
\text { (Guardant360 only) }\end{array}$ & 12 positive & $\begin{array}{c}8 \text { lung cancer: } \\
4 \text { Guardant } 360 \\
4 \text { InVisionFirst-Lung } \\
4 \text { solid tumorsc } \\
\text { Guardant } 360 \text { only }\end{array}$ \\
\hline
\end{tabular}

Abbreviations: ctDNA, circulating tumor DNA; LCD, local coverage determination; MAC, Medicare Administrative Contractor.

a Medicare coverage is provided by LCDs issued from 4 of 7 MACs. Medicare does not issue negative coverage policies, so we did not calculate a percentage of policies with positive coverage.

bBlue Cross Blue Shield Association-affiliated policies typically have 2 separate policy documents: 1 for lung cancer and 1 for all other cancers. These policies are counted individually.

cSolid tumors (12 types): non-small cell lung cancer, colorectal, breast, endometrial, gastric and gastroesophageal, gastrointestinal stromal tumor, melanoma, ovarian, pancreatic, prostate, thyroid, and chordoma. 
Table 2. Characteristics of 2019 Positive Coverage Policies by Payer

\begin{tabular}{|c|c|c|c|c|}
\hline Category & Private Payer Policy Characteristics & $\begin{array}{l}\text { Policies } \\
\text { n (\%) }\end{array}$ & Medicare Policy Characteristics & $\begin{array}{l}\text { Policies } \\
n\end{array}$ \\
\hline Total, n & & 28 & & 12 \\
\hline \multirow{5}{*}{ Cancers included } & Not defined & $11(39)$ & \multirow{4}{*}{ NSCLC (stage IIIB or IV) } & \\
\hline & Stage III or IV & $8(29)$ & & \\
\hline & Stage IIIB or IV & $3(11)$ & & \\
\hline & Advanced stage III or IV, or metastatic & $1(4)$ & & \\
\hline & Solid and hematologic malignancies & $4(14)$ & Solid tumors ${ }^{b}$ & 4 \\
\hline \multirow[t]{2}{*}{ ctDNA test covered } & $\begin{array}{l}\text { Single-gene EGFR analysis using cobas EGFR } \\
\text { Mutation Test v2, Guardant } 360 \text {, or } \\
\text { OncoBEAM }\end{array}$ & $12(43)$ & $\begin{array}{l}\text { Comprehensive genomic analysis using } \\
\text { InVisionFirst-Lung }\end{array}$ & 4 \\
\hline & $\begin{array}{l}\text { Comprehensive gene analysis using } \\
\text { Guardant } 360\end{array}$ & $10(36)$ & $\begin{array}{l}\text { Comprehensive gene analysis using } \\
\text { Guardant } 360\end{array}$ & 8 \\
\hline \multirow{2}{*}{ Monitoring covered } & $\begin{array}{l}\text { No prior testing and progression or } \\
\text { progression on TKls }\end{array}$ & $2(7)$ & $\begin{array}{l}\text { Not previously tested, or not responding } \\
\text { to EGFR TKls }\end{array}$ & 8 \\
\hline & At progression on EGFR TKls & $1(4)$ & $\begin{array}{l}\text { New primary cancer or different primary } \\
\text { cancer and untreated or not responding }\end{array}$ & 4 \\
\hline
\end{tabular}

Abbreviation: ctDNA, circulating tumor DNA; NSCLC, non-small cell lung cancer; TKI, tyrosine kinase inhibitor.

alncludes adenocarcinoma, large cell carcinoma, squamous cell carcinoma, and NSCLC not otherwise specified.

bSolid tumors (12 types): NSCLC, colorectal, breast, endometrial, gastric and gastroesophageal, gastrointestinal stromal tumor, melanoma, ovarian, pancreatic, prostate, thyroid, and chordoma.

\section{Discussion}

The overall trends in both private payer and Medicare coverage for ctDNA-based panel testing have been an increasing number of coverage policies, an increasing number of positive policies, and an increasing scope of coverage. We found that most positive coverage policies were very specifically written with defined clinical scenarios, although we also found that Medicare coverage policies were evolving to pan-cancer uses, signifying a significant shift in coverage frameworks.

As might have been expected given that the only FDA-approved ctDNA test is a single-gene test for NSCLC, we found that most (87\%) policies were for NSCLC and that nearly half $(47 \%)$ were for EGFR gene analysis. Furthermore, of those policies for NSCLC, most (79\%) only covered specifically named tests. One of the most interesting findings was in the case of EGFR gene analysis, in which $43 \%(12 / 28)$ payers stated that multigene panel tests (Guardant360 and OncoBEAM) would be a covered benefit for EGFR gene analysis only. Given that tests such as these are panel tests that evaluate multiple genes, the "limited" coverage decision may actually result in testing that is far more comprehensive than intended.

We found that BCBSA plans were heavily represented in our sample, but that such plans varied in their coverage policies. More than half $(64 \%)$ of the private payer policies were BCBSA plans, comprising $56 \%$ of the covered lives for all policies included. Of these, 22 policies provided coverage, representing $76 \%$ of all positive covered lives, and 25 policies did not provide coverage. Although the national BCBSA issued a technology assessment for "Circulating Tumor DNA and Circulating Tumor Cells for Cancer Management," ${ }^{18}$ BCBSA plans operate independently and each plan determines its own policies. ${ }^{10,19}$

One complexity of private payer coverage is that payers structure their policies in many ways, ranging from policies focusing on very specific clinical conditions, genes, and brand-named tests to broad policies that cover many conditions and generic tests. This wide variation reflects the fact that private payers vary greatly in how they develop and structure their coverage policies. ${ }^{20,21}$ Such variation increases the challenges in assessing and tracking coverage policies and may make it difficult for patients and providers to understand relevant policies.

An important finding was that Medicare coverage policies are evolving rapidly. Although there is no NCD policy explicitly providing coverage for ctDNA-based panel tests (other than for FDA-approved tests), coverage is provided through LCDs. The policy framework for Medicare LCDs is evolving from coverage of specific cancers to policies providing coverage of pan-cancer scenarios, which marks a significant change from earlier 
Table 3. Coverage Clinical Scenarios by Private Payer and Medicare

\begin{tabular}{|c|c|c|c|c|}
\hline Clinical Scenario & Cancer Type & Genes Included & Tests Included & \multirow{2}{*}{$\frac{\mathbf{n}(\%)}{4(14)}$} \\
\hline \multicolumn{4}{|c|}{ Private Payer Policy Characteristics } & \\
\hline 2 & NSCLC & EGFR gene analysis & $\begin{array}{l}\text { cobas EGFR Mutation Test v2, Guardant } 360 \text {, } \\
\text { or OncoBEAM only }\end{array}$ & $12(43)$ \\
\hline 3 & NSCLC & Genes not defined & Guardant 360 only & $8(29)$ \\
\hline 5 & NSCLC & EGFR gene analysis & Multigene panel not defined & $1(4)$ \\
\hline \multirow[t]{2}{*}{6} & NSCLC & Genes not defined & Multigene panel not defined & $1(4)$ \\
\hline & \multicolumn{2}{|r|}{ Medicare Policy Characteristics } & & $\mathbf{n}$ \\
\hline 7 & Solid tumors ${ }^{\mathrm{a}}$ & Genes not defined & Guardant $360^{\mathrm{b}}$ & 4 \\
\hline 8 & NSCLC & $E G F R, R O S, A L K, B R A F$ & Guardant 360 only & 4 \\
\hline
\end{tabular}

Abbreviation: NSCLC, non-small cell lung cancer.

aSolid tumors (12 types): NSCLC, colorectal, breast, endometrial, gastric and gastroesophageal, gastrointestinal stromal tumor, melanoma, ovarian, pancreatic, prostate, thyroid, and chordoma.

bother liquid biopsies will be covered for the same indications if they display similar performance in their intended used applications to Guardant360.

LCDs. Early LCDs were written in a very specific manner in which each one addressed a specific brand-named test in a particular cancer. For example, 4 MACs issued final LCD policies in 2018 for the Guardant360 ctDNA-based panel test for NSCLC, and the same 4 MACs issued final LCD policies in 2019 for the InvisionFirst ctDNA-based panel test for NSCLC. However, in early 2019, the same 4 MACs issued draft LCD policies that would provide pan-cancer coverage in 12 solid tumors. Two MACs have since issued a final LCD (effective February 3 and March 15, 2020). This coverage is still brand-name-specific given that it covers Guardant360, but the move toward pan-cancer coverage is significant, and the policy includes a provision that "other liquid biopsies will be covered for the same indications." $17 \mathrm{We}$ found in earlier studies that coverage of pan-cancer tests conflicts with many of the concepts of payer coverage frameworks, including that sequencing tests are considered a "bundle" of individual gene tests, which is counter to the one-marker-one-drug evaluation of medical necessity. ${ }^{21,22}$

We did not find any previous studies of payer coverage policies for ctDNA, but our results were consistent with other studies showing that coverage for sequencing tests is increasing in some clinical scenarios but that many tests are not covered. Our study evaluating payer

\begin{tabular}{|c|c|c|}
\hline Policy Structure (Focus of Policy) & $\begin{array}{c}\text { Private Payer } \\
\text { n (\%) }\end{array}$ & $\begin{array}{c}\text { Medicare }^{a} \\
\text { n (\%) }\end{array}$ \\
\hline Total, $\mathrm{n}$ & 73 & 12 \\
\hline Liquid biopsy overall & $26(36)$ & \\
\hline Third-party policyb & $12(16)$ & \\
\hline ctDNA NSCLC & $11(15)$ & $8(67)$ \\
\hline Tumor markers overall & $10(14)$ & \\
\hline Genetic testing overall & $6(8)$ & \\
\hline ctDNA solid tumors & $3(4)$ & $4(33)$ \\
\hline Test-specific_-Guardant360 & $1(1)$ & \\
\hline Expanded molecular testing panel overall & $1(1)$ & \\
\hline Other (eg, noncovered services, medical technologies database) & $3(4)$ & \\
\hline
\end{tabular}

Abbreviations: ctDNA, circulating tumor DNA; NSCLC, non-small cell lung cancer.

a Medicare does not issue negative coverage policies, so we did not calculate a percentage of policies with positive coverage.

bThird-party policies are those that are managed by a laboratory benefit management company (eg, Evicore or AIM). 
coverage policies for exome sequencing in pediatric patients with neurodevelopmental disorders found that $50 \%$ of private payers changed from no coverage to positive coverage between 2015 and $2017,{ }^{10}$ and our recent study of payer decision-making found that private payers were increasingly willing to cover such tests. ${ }^{13}$ However, our recent study of next-generation tumor sequencing coverage policies found that half of private payers were not providing coverage for such tests as of mid-2019. ${ }^{19}$

\section{Implications and Future Research}

Our results suggest that there is a conundrum between what the test actually evaluates, what the payer is willing to cover, and the information that the clinician receives and can use to guide clinical decisions. As noted earlier, several payers provide coverage for multigene tests but only allow the analysis of a single gene from those tests. For example, a clinician can order a particular test that interrogates 73 genes (eg, the Guardant360 test), but the payer will cover that test only for analysis of the single EGFR gene ( $43 \%$ of payers provide coverage in this manner), which leads to a report that may have results that are not covered by the payer but may still be used to inform the clinician's ability to properly manage the patient's cancer (reports are identical independent of payer coverage policy). In addition, limited policies, or having no policy, may burden clinicians with prior authorization requirements and appeals and thus delay or prevent access to testing. These issues will require further study. Another conundrum is that payer policies may not reflect what is found in reviews and guidelines. For example, ASCO and the College of American Pathologists published a review in 2018 that found no evidence of clinical utility and little evidence of clinical validity of ctDNA assays in early-stage cancer, treatment monitoring, or residual disease detection. ${ }^{7}$ The NCCN Clinical Practice Guidelines in Oncology (NCCN Guidelines) for NSCLC indicate that ctDNA testing can be considered when a patient is medically unfit for invasive tissue sampling or if, after pathologic confirmation of an NSCLC diagnosis, there is insufficient material for molecular analysis. ${ }^{23}$ In addition, studies that support clinical utility will likely result in greater consistency among coverage policies.

Another conundrum is that the technology used by some laboratories to detect ctDNA interrogates all cfDNA. The cfDNA contains germline DNA, which can provide insight into both related and nonrelated cancer susceptibility (eg, BRCA1/2 mutations in breast cancer vs $B R C A 1 / 2$ mutations in colon cancer). In addition to the ctDNA analysis, some laboratories provide the clinician with the results of cfDNA germline analysis for select genes. This fact presents a challenge given that these results may be unexpected and can confront both providers and patients with ethical and clinical dilemmas.

Although there has been a plethora of coverage for the use of ctDNA testing in NSCLC from both private payers and Medicare, the coverage has been primarily for treatment selection, with only $11 \%$ of payers with positive coverage including any monitoring indications. We expect that coverage may eventually be added for additional clinical indications, such as monitoring of minimal residual disease after definitive therapy or for detecting recurrence, although payers may be reluctant to adopt these indications because this would expand use to more extensive serial testing over time and thus may greatly increase costs.

\section{Limitations}

This study had several limitations. First, we did not include Medicaid policies, and the Canary Insights Database does not include all policies from all private payers in the United States, because it excludes self-insured and small payers. However, the Canary Insights Database includes $>200$ payers, and our analysis reflected coverage policies for $75 \%$ of the US population, including the 172 million individuals covered by private payers and the 75 million Medicare enrollees in 2019. Second, we were limited by the amount of information provided in the coverage policies by each payer, which were highly variable in their detail and clarity. We could not examine the actual decision-making process undertaken by individual payers. Third, published payer coverage policies do not necessarily reflect actual coverage or reimbursement for all "covered" tests, because plan purchasers can elect to exclude coverage for certain tests when purchasing plans for their employees. This is particularly true for self-insured groups, in which the insurer acts as a third-party administrator. Last, Medicare only issues positive coverage policies, which limited the comparison in both policy content and timing, given that the first Medicare policy was issued in 2018, whereas we included private payer policies from 2015 to 2019.

\section{Conclusions}

We found that coverage of ctDNA panel testing for cancer indications increased from 2015 to 2019. Two Medicare policies that have been finalized and additional policies that may be finalized in 2020 would provide a significant expansion of coverage to pan-cancer tests. Future research should continue to track coverage over time and evaluate the impact of coverage changes on test utilization. Given that genomic medicine is rapidly changing, payers and policymakers will need to continue to evolve policies to keep pace with emerging science and standards in clinical care.

\section{Acknowledgments}

We thank Bruce Quinn, MD, PhD (Bruce Quinn Associates LLC), for his insights, and Oliver Febbo, student intern (Urban School), 
for his assistance in data abstraction. Dr. Quinn and Mr. Febbo were not paid for their contributions.

Submitted October 11, 2019; accepted for publication January 29, 2020.

Author contributions: Study concept or design: Douglas, Phillips. Data acquisition, analysis, or interpretation: All authors. Manuscript preparation or critical revision: All authors.

Disclosures: Mr. Douglas has disclosed that he receives consulting fees from Illumina, Inc. Dr. Gray has disclosed that she has not received any financial consideration from any person or organization to support the preparation, analysis, results, or discussion of this article. Dr. Phillips has disclosed that she receives consulting fees from Illumina, Inc., and received consulting fees from Lexent Bio, Inc.

Funding: This work was supported by grants from the National Cancer Institute (R01 CA221870) and the National Human Genome Research Institute (U01 HG009599) to Dr. Phillips.

Disclaimer: The National Human Genome Research Institute and the National Cancer Institute had no role in the preparation, review, or approval of the manuscript or decision to submit the manuscript for publication.

Correspondence: Michael P. Douglas, MS, Department of Clinical Pharmacy, UCSF Center for Translational and Policy Research on Personalized Medicine, 3333 California Street, Room 420, Box 0613, San Francisco, CA 94143. Email: michael.douglas@ucsf.edu

\section{References}

1. Centers for Medicare \& Medicaid Services. Decision memo for next generation sequencing (NGS) for Medicare beneficiaries with advanced cancer (CAG-00450N). Accessed March 2, 2020. Available at: https:// www.cms.gov/medicare-coverage-database/details/nca-decision-memo. aspx?NCAld =290\&DocID =CAG-00450N\&bc=AAAAAAAAQAAA\&

2. Chin RI, Chen K, Usmani A, et al. Detection of solid tumor molecular residual disease (MRD) using circulating tumor DNA (ctDNA). Mol Diagn Ther 2019;23:311-331.

3. Corcoran RB, Chabner BA. Application of cell-free DNA analysis to cancer treatment. N Engl J Med 2018;379:1754-1765.

4. Duréndez-Sáez E Azkárate A, Meri M et al. New insights in non-small-cell lung cancer: circulating tumor cells and cell-free DNA. J Thorac Dis 2017; 9(Suppl 13):S1332-1345.

5. Karlovich CA, Williams PM. Clinical applications of next-generation sequencing in precision oncology. Cancer J 2019;25:264-271.

6. Marmarelis M, Thompson JC, Aggarwal C, et al. Emerging uses of circulating tumor DNA in advanced stage non-small cell lung cancer. Ann Transl Med 2017;5:380

7. Merker JD, Oxnard GR, Compton C, et al. Circulating tumor DNA analysis in patients with cancer: American Society of Clinical Oncology and College of American Pathologists joint review. J Clin Oncol 2018;36:1631-1641.

8. IJzerman MJ, Berghuis AMS, de Bono JS, et al. Health economic impact of liquid biopsies in cancer management. Expert Rev Pharmacoecon Outcomes Res 2018;18:593-599.

9. Oellerich M, Christenson RH, Beck J, et al. Plasma EGFR mutation testing in non-small cell lung cancer: a value proposition. Clin Chim Acta 2019; 495:481-486.

10. Douglas MP, Parker SL, Trosman JR, et al. Private payer coverage policies for exome sequencing (ES) in pediatric patients: trends over time and analysis of evidence cited. Genet Med 2019;21:152-160.

11. Phillips KA, Deverka PA, Trosman JR, et al. Payer coverage policies for multigene tests. Nat Biotechnol 2017;35:614-617.

12. Trosman JR, Weldon CB, Kelley RK, et al. Challenges of coverage policy development for next-generation tumor sequencing panels: experts and payers weigh in. J Natl Compr Canc Netw 2015;13: 311-318.
13. Trosman JR, Weldon CB, Slavotinek A, et al. Perspectives of US private payers on insurance coverage for pediatric and prenatal exome sequencing: results of a study from the Program in Prenatal and Pediatric Genomic Sequencing (P3EGS). Genet Med 2020;22:283-291.

14. Sheridan C. Investors keep the faith in cancer liquid biopsies. Nat Biotechnol 2019:37:972-974.

15. Slavin TP, Banks KC, Chudova D, et al. Identification of incidental germline mutations in patients with advanced solid tumors who underwent cell-free circulating tumor DNA sequencing [published online October 19, 2018] J Clin Oncol, doi:10.1200/JCO.18.00328:JCO1800328

16. Centers for Medicare \& Medicare Services. Advanced search. Accessed June 5, 2020. Available at: https://www.cms.gov/medicare-coveragedatabase/search/advanced-search.aspx

17. Centers for Medicare \& Medicaid Services. SUPERSEDED local coverage determination (LCD): MolDX: plasma-based genomic profiling in solid tumors (L38043). Accessed on June 5, 2020. Available at: https://www. $\mathrm{cms}$.gov/medicare-coverage-database/details/lcd-details.aspx? LCDId $=38043 \&$ ver $=3 \& b c=$ AAAAAAAAgAAA\&

18. Blue Cross Blue Shield Association. Evidence Street: Circulating tumor DNA and circulating tumor cells for cancer management (liquid biopsy). Accessed June 5, 2020. Available at: https://app.evidencestreet.com/

19. Trosman JR, Douglas MP, Liang SY, et al. Insights from a temporal assessment of increases in U.S. private payer coverage of tumor sequencing from 2015 to 2019. Value Health 2020;23:551-558.

20. Trosman JR, Van Bebber SL, Phillips KA. Coverage policy development for personalized medicine: private payer perspectives on developing policy for the 21-gene assay. J Oncol Pract 2010;6:238-242.

21. Trosman JR, Weldon CB, Gradishar WJ, et al. From the past to the present: insurer coverage frameworks for next-generation tumor sequencing Value Health 2018;21:1062-1068.

22. Trosman JR, Weldon CB, Douglas MP, et al. Payer coverage for hereditary cancer panels: barriers, opportunities, and implications for the Precision Medicine Initiative. J Natl Compr Canc Netw 2017;15:219-228.

23. Ettinger DS, Wood DE, Aggarwal C, et al. NCCN Clinical Practice Guidelines in Oncology: Non-Small Cell Lung Cancer. Version 3.2020. Accessed April 24, 2020. To view the most recent version, visit NCCN.org. 
Supplemental online content for:

\section{Private Payer and Medicare Coverage for Circulating Tumor DNA Testing: A Historical Analysis of Coverage Policies From 2015 to 2019}

Michael P. Douglas, MS; Stacy W. Gray, MD; and Kathryn A. Phillips, PhD

J Natl Compr Canc Netw 2020;18(7):866-872

eTable 1: Variables Coded for Private and Medicare Policies

eAppendix 1: Definitions

eAppendix 2: Canary Insights Database

eAppendix 3: Policy Search Validation

eAppendix 4: Description of ctDNA Tests Used in Any ctDNA Panel 
eTable 1. Variables Coded for Private and Medicare Policies

\begin{tabular}{|c|c|c|}
\hline Variable name & Variable Definition & Variable Details/Coding \\
\hline Policy unique ID & Automatic unique number & Fill down sequentially \\
\hline Payer name & Payer full name & (eg, Aetna) \\
\hline Policy name & Official name of policy & (Copy from Policy Source Information Table) \\
\hline Policy type & Type of policy focus & $\begin{array}{l}\text { Genetic testing overall } \\
\text { Tumor markers overall } \\
\text { Expanded molecular testing panel overall } \\
\text { Liquid biopsy overall (ctDNA and circulating } \\
\text { tumor cells) ctDNA NCSLC } \\
\text { ctDNA solid tumors } \\
\text { Third-party policy (eg, AIM, eviCore) } \\
\text { Test-specific (eg, Guardant) } \\
\text { Other }\end{array}$ \\
\hline Policy from third party? & $\begin{array}{l}\text { Was this policy adapted by the payer from a } \\
\text { third party (eg, eviCore)? }\end{array}$ & Yes (note third party)/No \\
\hline Most recent policy date & Date of policy & (MM/DD/YYYY) \\
\hline Policy identified in search & Yes/No & Yes/No \\
\hline Coverage & Yes/No & Yes/No \\
\hline How coverage worded & $\begin{array}{l}\text { What is covered in summary (cancer type, } \\
\text { genes, test) }\end{array}$ & Summarize from copy/paste \\
\hline Cancers included & $\begin{array}{l}\text { Covered cancers (eg, lung cancer, pan-cancer, } \\
\text { solid tumors) }\end{array}$ & Copy/Paste from policy \\
\hline Test covered for monitoring & Yes/No & Yes/No \\
\hline How is monitoring covered? & Short description if applicable & Copy/Paste or summarize \\
\hline What ctDNA testing is covered & $\begin{array}{l}\text { Names of tests covered, if specified, or } \\
\text { "general ctDNA or multigene tests" }\end{array}$ & Copy/Paste from policy \\
\hline Not covered language & Words used to describe what is not covered & Copy/Paste from policy \\
\hline Covered clinical scenario & $\begin{array}{l}\text { For which clinical indications is next-generation } \\
\text { tumor sequencing considered medically } \\
\text { necessary (eg, all solid tumors, advanced lung } \\
\text { cancer, hematologic cancers) }\end{array}$ & Copy/Paste from policy \\
\hline
\end{tabular}




\section{eAppendix 1. Definitions}

Cell-Free DNA (cfDNA) are degraded DNA fragments released to the blood plasma. cfDNA can be used to describe various forms of DNA freely circulating the bloodstream, including circulating tumor DNA (ctDNA) and cell-free fetal DNA (cffDNA). Elevated levels of cfDNA are observed in cancer, especially in advanced disease.

Circulating Tumor DNA (ctDNA) is tumor-derived fragmented DNA in the bloodstream that is not associated with cells. ctDNA should not be confused with cell-free DNA (cfDNA), a broader term which describes DNA that is freely circulating in the bloodstream, but is not necessarily of tumor origin. ctDNA originates directly from the tumor or from circulating tumor cells (CTCs). Because ctDNA may reflect the entire tumor genome, it has gained traction for its potential clinical utility; "liquid biopsies" in the form of blood draws may be taken at various time points to monitor tumor progression throughout the treatment regimen.

Circulating Tumor Cells (CTCs) are whole tumor cells shed into the vasculature from a primary tumor and are carried around the body in the blood. CTCs may constitute seeds for subsequent growth of additional tumors (metastasis) in distant organs, a mechanism that is responsible for most cancer-related deaths. We do not examine coverage policies for CTC testing in this study.

Liquid Biopsy is a term used to refer to the analysis of ctDNA or CTCs from blood, urine, or other fluid.

\section{eAppendix 2. Canary Insights Database}

The Canary Insights Database (http://canaryinsights.com/) is a medical policy library containing $>40,000$ medical policies from commercial payers and links to public payer policies. Canary Insights continuously mines coverage and reimbursement changes for therapy and products. Canary Insights performs a daily search using a proprietary search engine on $>200$ payers and related healthcare sites, including Medicare, Medicaid, and hundreds of commercial insurance companies, and is updated on a daily basis. It does not include actual public payer coverage policies but does link to the Centers for Medicare \& Medicaid Services website. Canary Insights provides a search engine that allows one to use keywords and select payers to identify private payer policies. The most current version of the policy is accessible via a link to the actual payer website, and past versions are provided in a PDF format. Individual Policy Data are validated as a direct product from the payer's website (via a link or a PDF of the previous policy) and not abstracted/curated by Canary Insights (ie, data were not abstracted by Canary Insights).

\section{eAppendix 3. Policy Search Validation}

We used an iterative approach to identifying the best keywords to identify ctDNA-based panel tests. Specifically, we used a previous sample of 2015-2018 ctDNA policies and noted their titles. We found that nearly all included the terms "liquid biopsy" or "circulating tumor DNA." We also found that some policies were written with general terminology in their titles, such as "expanded cancer panels" or "noncovered services." We validated our terms by confirming that we did not find any instances of ctDNA-based coverage determinations within other policies (eg, Hereditary Cancer Genetic Testing) or that the policies we did identify (and subsequently included) using our search terms did not refer to additional policies that may include a ctDNA-based panel test coverage determination. For example, in all policies that referred to additional policies, we verified that they referred to policies that did not contain further information or determinations on ctDNA-based panel test coverage.

\section{eAppendix 4. Description of ctDNA Tests Used in Any ctDNA Panel}

NOTE: Tests that were named in any circulating tumor DNA (ctDNA)-based panel test coverage policy are described below. Inclusion of a named test that interrogates a single gene below should not construe coverage policies for single gene tests were included in this study.

\section{cobas EGFR Mutation Test v2}

Manufacturer: Roche

Cancer Type: Non-small cell lung cancer (NSCLC)

Test Sample: Cell-free DNA (cfDNA)

FDA Approval: Yes

Number of Genes Analyzed: 1 (EGFR)

Test Purpose (per manufacturer): 
- The cobas EGFR Mutation Test v2 is a real-time PCR test for the qualitative detection of defined mutations of the EGFR gene in patients with NSCLC. Defined EGFR mutations are detected using DNA isolated from formalin-fixed paraffinembedded tumor tissue or cfDNA from plasma derived from EDTA anticoagulated peripheral whole blood.

- The test is intended to aid in identifying patients with NSCLC whose tumors have defined EGFR mutations and for whom safety and efficacy of a drug have been established as follows: Tarceva (erlotinib), exon 19 deletions and L858R; Tagrisso (osimertinib), T790M. Drug safety and efficacy have not been established for the following EGFR mutations also detected by the cobas EGFR Mutation Test v2: Tarceva (erlotinib), G719X, exon 20 insertions, T790M, S768I, and L861Q; Tagrisso (osimertinib), G719X, exon 19 deletions, L858R, exon 20 insertions, S768I, and L861Q.

\section{Guardant360}

Manufacturer: Guardant Health

Cancer Type: NSCLC, pan-cancer

Test Sample: ctDNA

FDA Approval: No

Number of Genes Analyzed: 73 genes, 23 indels, 18 amplifications, 6 fusions

Test Purpose (per manufacturer) ${ }^{1}$ :

- Guardant360 is a laboratory test, which is performed with a blood sample. Patients are suitable for this test if they have a solid tumor in an advanced stage (stage III or IV) and are planned to be treated with targeted drugs.

- Before first-line treatment: Get ahead of the challenges of tissue testing in advanced NSCLC by utilizing Guardant360 to guide first-line treatment decisions

- At progression: Obtain genomic information on $>70$ genes relevant across multiple solid tumors including MSI-high to help find pan cancer therapies and clinical trials. Furthermore, in case of progression or when there is a tumor that is not responding to therapy after some time, it can be examined if probably further mutations came along, which are relevant for therapy planning. It can happen during a cancer therapy that a tumor develops strategies, so-called resistance mechanisms, via further mutations to avoid therapy and continue to spread.

- Monitoring and aftercare: Guardant360 can be applied for the monitoring and aftercare of a cancer therapy as well. The liquid biopsy can be used to not only detect present mutations but also determine the level of present ctDNA in general. Therefore, whether a tumor responds to a corresponding therapy with targeted drugs, chemotherapeutics, or any other cancer therapy it be periodically observed without a big intervention. When a high level of tumor cells is present in the body, there is also a higher level of ctDNA in the blood. If a tumor responds to a therapy and decreases, the level of ctDNA in the blood also decreases or disappears.

\section{InVisionFirst-Lung}

Manufacturer: Inivata

Cancer Type: NSCLC

Test Sample: ctDNA

FDA Approval: No

Number of Genes Analyzed: 36

Test Purpose (per manufacturer) ${ }^{2}$ :

- InVisionFirst-Lung is a qualitative laboratory-developed test that uses targeted advanced sequencing technology to detect single nucleotide variants, copy number variants, insertions and deletions (indels), and structural variants in selected genes from DNA isolated from plasma samples from patients with NSCLC. The test is intended to aid clinicians in making treatment decisions for patients with NSCLC.

\section{OncoBEAM for Lung Cancer-1}

Manufacturer: Sysmex Inostics

Cancer Type: NSCLC

Test Sample: ctDNA

FDA Approval: No

Number of Genes Analyzed: 1 (EGFR)

Test Purpose (per manufacturer) ${ }^{3}$ :

- The highly sensitive BEAMing technology, a liquid biopsy with OncoBEAM, requires only a simple blood draw to access tumor DNA and provide a real-time view of a tumor's mutation status.

- The test analyzes the EGFR sensitizing mutations del19, L858R, and L861Q, and resistant mutations T790M, C797S.

- NSCLC tumors with sensitizing EGFR mutations have increased sensitivity to tyrosine kinase inhibitors (TKIs). Patients who initially respond to TKI therapy frequently relapse, with $60 \%$ of those on TKIs developing resistance due to the EGFR T790M mutation. 
- OncoBEAM EGFR can identify the T790M mutation in patients whose disease has progressed on first-line TKI therapy, delivering rapid results and avoiding the need for a repeat tissue biopsy.

\section{OncoBEAM for Lung Cancer-2}

Manufacturer: Sysmex Inostics

Cancer Type: NSCLC

Test Sample: ctDNA

FDA Approval: No

Number of Genes Analyzed: 3 (EGFR, KRAS, BRAF)

Test Purpose (per manufacturer) ${ }^{3}$ :

- The highly sensitive BEAMing technology, a liquid biopsy with OncoBEAM, requires only a simple blood draw to access tumor DNA and provide a real-time view of a tumor's mutation status.

- The test analyzes the EGFR sensitizing mutations del19, L858R, and L861Q, and resistant mutations T790M, C797S; KRAS codons 12, 13, and 61; and BRAF V600E.

- NSCLC tumors with sensitizing EGFR mutations have increased sensitivity to TKIs. Patients who initially respond to TKI therapy frequently experience relapse, with $60 \%$ of those on TKIs developing resistance due to the EGFR T790M mutation.

- OncoBEAM EGFR can identify the T790M mutation in patients who have experienced disease progression on first-line TKI therapy, delivering rapid results and avoiding the need for a repeat tissue biopsy.

- OncoBEAM KRAS can identify multiple KRAS mutations, providing a more complete picture of a patient's condition to rapidly inform critical therapy decisions. KRAS mutations occur in 25\% of NSCLC cases. Independent of therapy, KRAS mutations can indicate poor survival rates compared with tumors without KRAS mutations.

- OncoBEAM BRAF tests for the BRAFV600E mutation, helping doctors to decide whether to initiate first-line therapy as soon as possible. BRAF mutations are detected in approximately $3 \%$ of patients with NSCLC. ${ }^{4}$ In 2017 , the NCCN Clinical Practice Guidelines in Oncology for NSCLC ${ }^{4}$ were updated to include BRAFV600E mutation testing for all newly diagnosed patients to inform appropriate administration of first-line therapy, which now includes BRAF-targeted therapies.

\section{References}

1. Guardant Health. Guardant360. Accessed June 5, 2020. Available at http://www.guardant360.com/

2. Inivata. InVisionFirst-Lung. Accessed June 5, 2020. Available at: https://www.inivata.com/invisionfirst-lung/

3. Sysmex Inostics, Inc. OncoBEAM for Lung Cancer. Accessed June 5, 2020. Available at: https://www.oncobeam.com/healthcare-providers/lung-cancer

4. Ettinger DS, Wood DE, Aisner DL, et al. NCCN Clinical Practice Guidelines in Oncology: Non-Small Cell Lung Cancer. Version 7.2017. To view the most recent version of these guidelines, visit NCCN.org. 